\title{
$5 \quad$ Feststellung des Todes und Mitteilung an die Koordinierungsstelle
}

\author{
Eckhard Rickels und Stefanie Förderreuther
}

Im Transplantationsgesetz (TPG) ist geregelt, dass

„die Entnahme von Organen oder Geweben nur zulässig ist, wenn der Tod des Organ- oder Gewebespenders nach Regeln, die dem Stand der Erkenntnisse der medizinischen Wissenschaft entsprechen, festgestellt ist“. (TPG \$3, Abs. 1 Satz 2)

Vor jeder Entnahme eines Organs oder von Gewebe muss der endgültige, nicht behebbare Ausfall der Gesamtfunktion des Großhirns, des Kleinhirns und des Hirnstamms festgestellt werden (TPG $₫ 3$ Abs. 2. Satz 2). Dabei obliegt es der Bundesärztekammer gemäß $\S 16$ TPG, den Stand der Erkenntnisse der medizinischen Wissenschaft für die Todesfeststellung durch Verfahrensregeln zur Feststellung des endgültigen, nicht behebbaren Ausfalls der Gesamtfunktion des Großhirns, des Kleinhirns und des Hirnstamms einschließlich der dazu jeweils erforderlichen ärztlichen Qualifikation festzulegen.

\subsection{Formale und praktische Besonderheiten für den Nachweis des irreversiblen Hirnfunktionsausfalls (IHA)}

Für die Praxis heißt das, dass jede Todesfeststellung durch Nachweis des nicht behebbaren Ausfalls der Gesamtfunktion von Großhirn, Kleinhirn und Hirnstamm gemäß der jeweils gültigen Richtlinie der Bundesärztekammer erfolgen muss. Dies stellt sicher, dass der Nachweis des irreversiblen Hirnfunktionsausfalls (IHA) dem aktuellen wissenschaftlichen Erkenntnisstand 
entspricht. In Deutschland ist kein Fall bekannt, bei dem die Feststellung des irreversiblen Hirnfunktionsausfalls bei korrekter Anwendung der Richtlinie gemäß $\$ 16$ Abs. 1 S. 1 Nr. 1 TPG unzutreffend gewesen wäre (Deutsches Ärzteblatt 2018). Derzeit ist die Vierte Fortschreibung der Richtlinie vom 30.1.2015 gültig.

Die Feststellung des IHA erfolgt nach speziellen, reglementierten Verfahren, die über die grundlegenden ärztlichen Kompetenzen für die Feststellung des Todes durch eine sorgfältige Leichenschau hinausgehen. Dies erfordert besondere intensivmedizinische Erfahrung, da der Tod unter maschineller Beatmung und bei aufrecht erhaltener Kreislauffunktion festgestellt wird. Durch den Nachweis des IHA wird ein ebenso sicheres Todeszeichen festgestellt, wie durch den Nachweis eines anderen sicheren Todeszeichens, wie z.B. von Totenflecken oder einer Leichenstarre. Eine Rückkehr zum Leben in körperlich-geistiger Einheit ist nicht mehr möglich, da das Gehirn hierfür die unverzichtbare körperliche Grundlage darstellt. Grundsätzlich ist der IHA auch dann immer eingetreten, wenn ein anderes sicheres Todeszeichen festgestellt wurde.

Der Verdacht auf die Entwicklung eines IHA ergibt sich bei schweren Hirnschädigungen, wenn sich der neurologische Befund zunehmend verschlechtert und ein Anstieg des intrakraniellen Drucks nicht mehr beherrscht werden kann.

Bei Patienten mit so schweren Hirnschädigungen muss neben der individuellen Beurteilung der Gesamtprognose auch die Möglichkeit einer Organspende bei den weiteren intensivmedizinischen Therapieentscheidungen bedacht werden. Ist die maximale Intensivbehandlung für den Betroffenen nicht Erfolg versprechend oder eine Fortführung der Intensivbehandlung nicht dem Patientenwillen entsprechend, ist gemäß den gültigen gesetzlichen Grundlagen zu prüfen, ob der Betroffene nach ärztlicher Beurteilung als Organspender in Betracht kommt.

Um einen potentiellen Spender zu erkennen, hilft die Richtlinie Spendererkennung der BÄK. Darüber hinaus gibt es die Möglichkeit prinzipielle Fragen zur Spendereignung ohne Übermittlung persönlicher Daten des Betroffenen mit der Koordinierungsstelle zu besprechen und bei Bedarf auch Unterstützung für die Feststellung des IHA z.B. durch Hinzuziehen eines Konsilarztes oder durch ergänzende Laboruntersuchungen anzufordern.

Besteht der Verdacht, dass der IHA in absehbarer Zeit eintritt oder bereits eingetreten ist, ist in Abhängigkeit von der klinischen Befundentwicklung eine IHA Diagnostik durchzuführen und der Tod unverzüglich bei der zuständigen Koordinierungsstelle mitzuteilen (TPG $₫$ ga). 


\subsection{Ablauf der Todesfeststellung}

Der Nachweis des IHA erfolgt obligat durch mindestens zwei Ärzte, die unabhängig voneinander zunächst überprüfen, ob die Voraussetzungen für einen Hirnfunktionsausfall erfüllt sind, dann durch eigenhändige Untersuchung das Fehlen der Hirnfunktionen nachweisen und schließlich den Nachweis der Irreversibilität der Ausfallsymptome führen. Beide Untersucher müssen Fachärzte mit mehrjähriger intensivmedizinischer Erfahrung sein. Einer der Untersuchenden muss Neurologe oder Neurochirurg sein. Bei Kindern bis zum 14. Lebensjahr muss ein Untersucher auch Facharzt für Kinder- und Jugendmedizin sein. Die an der Feststellung des IHA beteiligten Ärzte dürfen weder an der Entnahme noch an der Übertragung der Organe oder Gewebe des Spenders beteiligt sein und sie dürfen auch nicht Weisungen eines Arztes unterstehen, der an diesen Maßnahmen beteiligt ist.

Grundsätzlich erfordert das Prüfen der Voraussetzungen für die Untersucher den Ausschluss von reversiblen Funktionsstörungen, von Faktoren, die den klinischen Untersuchungsbefund verschleiern könnten, und den Nachweis einer Diagnose, die zwischen primären supratentoriellen, primären infratentoriellen, sekundären und kombinierten Hirnschädigungen differenziert.

\section{Die klinische Untersuchung erfordert den Nachweis des tiefen Komas, des Ausfalls aller Hirnnervenreflexe und der Vitalfunktio- nen des Hirnstamms.}

Die Irreversibilität der klinischen Ausfallsymptome wird in Abhängigkeit von der Art der Hirnschädigung (primär vs. sekundär) und deren Lokalisation (supratentoriell vs. infratentoriell) durch erneute klinische Untersuchungen nach definierten Beobachtungszeiten oder durch zusätzliche apparative Verfahren (z.B. EEG, Verfahren zum Nachweis des Perfusionsstillstandes) belegt. Für die Erbringer von apparativen Zusatzuntersuchungen sind ebenfalls spezielle Qualifikationsanforderungen und Verfahrensanweisungen für die Durchführung der Untersuchungen in der Richtlinie definiert.

Da sich bei Neugeborenen und Säuglingen vor Vollendung des zweiten Lebensjahres die Reifung des Gehirns und die Entwicklung der Ausfallsymptome auf Grund der anatomischen Besonderheit der noch offenen Schädelnähte und Fontanellen von der eines älteren Menschen unterscheiden, sind für die Todesfeststellung vor Vollendung des zweiten Lebensjahres zusätzliche besondere Anforderungen für die IHA Diagnostik vorgeschrieben. Bei 
unreifen Frühgeborenen und bei Anencephalgie ist die Richtlinie grundsätzlich nicht anwendbar.

Die Ergebnisse aller Untersuchungsschritte werden von den Untersuchern in Protokollbögen entsprechend der Anlage 1 der Richtlinie der BÄK schriftlich festgehalten. Der IHA ist festgestellt, wenn alle erforderlichen Untersuchungen mit übereinstimmenden Ergebnissen zweifelsfrei dokumentiert sind. Als Todeszeitpunkt wird das Datum mit der Uhrzeit festgehalten, zu dem die Untersuchungen abgeschlossen waren. Es unterzeichnen die beiden abschließenden Untersucher.

\subsection{Meldepflicht}

Gemäß s 9a TPG müssen Patienten, die nach ärztlicher Einschätzung als Spender in Betracht kommen, spätestens mit der Feststellung des Todes der Koordinierungsstelle gemeldet werden. Der zuständige Transplantationsbeauftragte ist dafür verantwortlich, dass die Entnahmekrankenhäuser dieser Verpflichtung nachkommen. Es obliegt dem Transplantationsbeauftragten zudem bei allen Todesfällen mit primärer oder sekundärer Hirnschädigung die Gründe für eine nicht erfolgte Feststellung oder eine nicht erfolgte Meldung des IHA in jedem Einzelfall auszuwerten (TPG $₫ 9 b$ ).

\subsection{Dokumentation und amtliche Todesbescheinigung}

Der ermittelte Todeszeitpunkt ist auch in den amtlichen Leichenschauschein einzutragen. Die amtliche Todesbescheinigung erfordert zudem die Angabe, ob Anhaltspunkte für einen nicht natürlichen Todesfall vorliegen ( $\$ 3$, Abs. 1 Nds. Gesetz über das Leichen-, Bestattungs- und Friedhofswesen). Bei Verdacht auf eine nicht natürliche Todesursache (z.B. Unfall, Fremdverschulden) ist die Polizei bzw. Staatsanwaltschaft unverzüglich zu informieren. Dies gilt auch im Falle einer potenziellen Organspende. Die Koordinierungsstelle kann die Kliniken bei der Kontaktvermittlung unterstützen. Die Behörden sind im Fall einer potenziellen Organspende gehalten, rasch zu entscheiden, ob eine Explantation erfolgen kann und ob ein Gerichtsmediziner aus Ermittlungsgründen bei der Explantation zugegen sein muss.

\section{Literatur}

Brandt SA, Angstwurm H (2018) Bedeutung des irreversiblen Hirnfunktionsausfalls als sicheres Todeszeichen. Deutsches Ärzteblatt. 12. Oktober 2018. Ig. 115, Heft 41, S. 675 
5.4 Dokumentation und amtliche Todesbescheinigung

Bundesärztekammer (2020) Richtlinie gemäß $\oint 16$ Abs. 1 S. 1 Nr. 3 TPG zur ärztlichen Beurteilung nach $\S$ ga Abs. 2 Nr. 1 TPG (RL BÄK Spendererkennung). URL: https://www.bundesaerztekammer.de/ fileadmin/user_upload/downloads/pdf-Ordner/RL/RiliSpendererkennung_2020-09-01.pdf (zuletzt abgerufen am 08.10.2021)

Deutsches Ärzteblatt (2015) Richtlinie gemäß $₫ 16$ Abs. 1 S. 1 Nr. 1 TPG für die Regeln zur Feststellung des Todes nach $\S 3$ Abs. 1 S. 1 Nr. 2 TPG und die Verfahrensregeln zur Feststellung des endgültigen, nicht behebbaren Ausfalls der Gesamtfunktion des Großhirns, des Kleinhirns und des Hirnstamms nach $\S 3$ Abs. 2 Nr. 2 TPG, Vierte Fortschreibung. 30. März 2015. DOI: 10.3238/ arztebl.2015.rl_hirnfunktionsausfall_01

Deutsches Ärzteblatt (2018) Erklärung zur Bedeutung des irreversiblen Hirnfunktionsausfalls als sicheres Todeszeichen. 12. Oktober 2018. Ig. 115, Heft 41, S. A1836 